\title{
Segmentation Method of Skin MRI High Resolution in vivo
}

\section{Type of article: Original article.}

\author{
Rachida Zegour ${ }^{1}$,Ahror Belaid ${ }^{2}$, Douraied Ben Salem ${ }^{3,4}$ \\ ${ }^{1}$.Ph.D. student at Medical Computing Laboratory (LIMED), Faculty of Exact Sciences,University \\ of Abderrahmane Mira of Bejaia, Algeria. \\ 2. Assistant professorat Medical Computing Laboratory (LIMED), Faculty of Exact \\ Sciences,University of Abderrahmane Mira of Bejaia, Algeria. \\ ${ }^{3}$. Professor at INSERM UMR 1101 Laboratory of Medical Information Processing (LaTIM), 5 \\ avenue Foch, 29200 Brest, France. \\ ${ }^{4}$.Professor at Neuroradiology Department, CHRU-Brest, boulevard Tanguy-Prigent, \\ 29609 Brest, France.
}

\begin{abstract}
:
Background: Recently, Magnetic Resonance Imaging (MRI) has been used in clinical application as non-invasive medical modality; it is rarely used to study the anatomy physiological, and biochemical of the skin, in spite of its very attractive modality for skin imaging. It makes an ideal imaging modality of unique soft tissue contrast to study the skin anatomy and to observe the different skin's layers. However, MRI provides a big data with high quality. The analysis of these data requires computerized methods to help clinicians and to improve diagnosis. Several image processing methods have been used by doctors to facilitate qualitative diagnosis, segmentation is one of these methods used in clinical applications in order to understand medical data and extract useful information. This study aims to use the segmentation method in order to observe the anatomy of skin layers before and after applying moisturizer.

Methods: We will classify segmentation approaches for MRI data into three basics classes: Edge based segmentation; Region based segmentation, and Thresholding segmentation. Then we will briefly describe Fuzzy C-means Clustering method, which is used to segment our MR data represented by a sample of thirty-five (35) healthy.

Results: We have observed the skin's layers before and after applying moisturizer topic of the feet as a result of the FCM segmentation method.

Our study showed that FCM is an efficient Algorithm used for medical images, because of its fuzzy nature; also it gives images segmentation results with different classes of skin layers before and after the application of moisturizer topic.

Conclusion: MRI is an attractive modality to study the human skin; it makes an ideal observation of the different skin layers in vivo. However, the segmentation of MRI data by FCM clustering is a computerized method to help clinicians in order to study the skin anatomy.
\end{abstract}

Keywords: MRI High Resolution, segmentation, FCM.

Corresponding author: Rachida Zegour, Medical Computing Laboratory (LIMED), Faculty of Exact Sciences, University of Abderrahmane Mira of Bejaia, Algeria Email: zegourr@ gmail.com

Received: Mai 30, 2018, Accepted: September 02, 2018, English editing: September 28, 2018, Published: September 30, 2018.

Screened by iThenticate.@2017-2018 KNOWLEDGE KINGDOM PUBLISHING. 


\section{Introduction}

Over the last years, Medicine has used many image modalities in order to make diagnostic easier. Magnetic Resonance Imaging (MRI) is one of non-invasive modalities which uses the distribution of water in human body in order to produce medical images.

MRI modality provides a big data with high quality, the analysis of these data to extract important information manually becomes a complex task for clinicians, it is time consuming and it may cause errors because of the large and various studies. However, MR data analysis requires a set of computerized methods to improve diagnosis and make it easy [2].

Nowadays, many computerized methods assist doctors for MR data diagnostic. Segmentation method is most used in MR data processing for many applications in order to understand medical data and extract useful information.

Hence, MRI modality is rarely used in clinical application to study the anatomy physiological, and biochemical of the skin [1], but is a very attractive modality for skin imaging. It makes an ideal imaging modality of unique soft tissue contrast to study the skin anatomy and to observe the different skin's layers [6].

Skin covers the total area of the human body, it is composed of three layers [3]: (1) Epidermis: the outermost layer of skin provides a waterproof barrier, which is composed of approximately $95 \%$ of keratinocytes, with four layers: basal, spinous, granular and corneum layers. These layers are undergoing continuous transformation and form the remaining 5\% [4]. (2) Dermis: is beneath the epidermis, which is composed of fibroblasts, dermal dendritic cells, mastocytes and macrophages $(70 \%)$. Other components include collagen, elastic fibers and air follicles. The dermis is grouped into two categories: papillary dermis and reticular dermis [4]. (3) Fat: The deeper subcutaneous tissue is made of fat and connective tissue [3].

Water distribution in human skin plays a key role indifferent skin functions, such as thermoregulation, barrier function...etc. [5]. Magnetic Resonance Imaging (MRI) uses magnetic proprieties of some atoms like Hydrogen $\left({ }^{1} \mathrm{H}\right)$, Sodium $\left({ }^{23} \mathrm{Na}\right)$, Carbon $\left({ }^{13} \mathrm{C}\right)$..etc. As the human body is made of $70 \%$ water $\left(\mathrm{H}_{2} \mathrm{O}\right)$, MRI in clinical applications, uses magnetic properties of Hydrogen atoms to produce medical images [7].

This paper will address a medical challenge of in vivo high-resolution skin MR imaging, our data is skin MR images has been investigated using 3-Tesla scan. With high resolution to make the promising of skin in vivo approaches possible, and also allows observing the anatomical and the physiological properties of the skin with fine exploration [1]. Our data have been processed using the segmentation method in order to observe the skin anatomy of the feet before applying moisturizer topic and one hour after that. We will also review in this paper the methods of MR images segmentation, then; we will present their classification with a detailed description of Fuzzy C-means Clustering (FCM) method. Furthermore, we will give some related works used FCM algorithm with MRI images. Then we will describe our study's sample, and come up to discuss the results. Finally, we will conclude the review by a conclusion. 


\section{Methods}

Segmentation is one of the most used methods to process images with several approaches. In this paper, we present a categorization of three classes for medical image methods (see figure 1) [8]: (a) Edge-based segmentation: consists of finding boundaries between regions based on detecting sharp and local changes in the intensity value, with three interested characteristics: edge, line and isolated point. (b) Region-based segmentation: based on a set of intensity attributes value to find the similar region directly. This approach involves many methods such as region growing, split, merge and clustering method. (c) Thresholding method: is one of the most applied approaches in image processing, because of its properties, simplicity, and its speed of computation. It classifies each pixel of image according to the specific threshold value.

Other approved methods have been developed such as morphological watersheds, active contours and level set method. The following diagram represents the classification methods of segmentation:

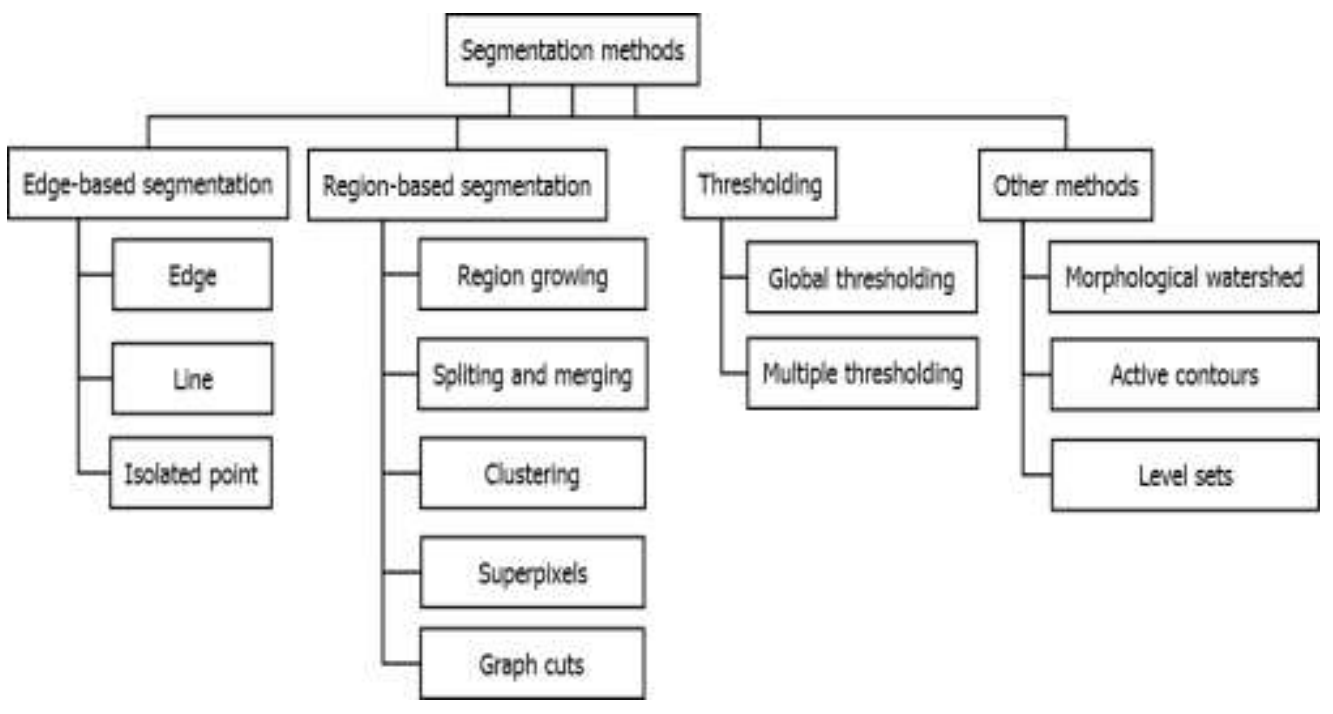

Figure 1: Classification of segmentation methods

Fuzzy C-means Clustering (FCM) is a clustering method used to segment images, it assigns similar pixels into the same class using fuzzy membership.

Let $\mathrm{X}=\left(\mathrm{x}_{1}, \mathrm{x}_{2}, \ldots \ldots, \mathrm{x}_{\mathrm{n}}\right)$ represents an image with $\mathrm{n}$ pixels, which is splitted into $\mathrm{C}$ clusters. Where, each $\mathrm{x}_{\mathrm{i}}$ represents a set of pixel features. FCM algorithm minimizes the following objective function [11]:

$\mathrm{J}=\sum_{j=1}^{N} \sum_{l=1}^{C} U_{i j}\left\|x_{j}-v_{i}\right\|^{2}$

Where, $u_{i j}$ is the membership of pixel $x_{j}$ in the $i^{\text {th }}$ cluster, $v_{i}$ represents the $i^{\text {th }}$ cluster center, $\|$.$\| is a norm metric, and m$ is a parameter which defines the fuzziness of the resulting clusters.

FCM algorithm assigns a high membership to the pixels when they are close to the centroid of the cluster and a low membership if not, in order to minimize the objective function J. The probability value of pixels depends solely on the distance between the pixel and each individual cluster center. FCM algorithm starts with an initial guess for each cluster center, then update membership and cluster centers for each iteration as follows [11]: 


$$
\begin{gathered}
U_{i j}=\frac{1}{\sum_{k=1}^{c}\left(\frac{\left\|x_{j}-v_{j}\right\|^{2}}{\left\|x_{j}-v_{k}\right\|}{ }^{/(m-1)}\right)} \\
v_{i}=\frac{\sum_{j=1}^{n} u_{i j x_{j}}^{m}}{\sum_{j=1}^{n} u_{i j}}
\end{gathered}
$$

When the changes in the membership function or the cluster center at two successive iteration steps are constant, FCM algorithm converges to a solution with $\mathrm{v}_{\mathrm{i}}$ representing a saddle point of the objective function [11].

FCM is used for medical images because of its fuzzy nature, where one pixel can belong to more than one cluster according to different probability value, and with a higher performance than crisp methods [9]. For example, some developers [11-13] used spatial FCM algorithm for MRI data to improve the robustness of the conventional algorithm. Others [14] used FCM algorithm to segment MRI images. Fuzzy C-means Clustering (FCM) method has two problems: The first one is its less performance with imaging noise, and the second is the Euclidean distance which is used [10]. Because of these problems, many variations to the FCM have been developed like Bias-Corrected Fuzzy C-Means (BCFCM), Possibilistic Fuzzy C-Means (PFCM) ... etc [9].

\section{Results and discussion}

Our data are the results of a study conducted between September 2014 and August 2015 in the research center at the university Hospital in Brest, France [1]. The study performed an MRI of the feet before and after applying moisturizer topic. The study's sample was thirty-five healthy volunteers comprising 17 women and 18 men with an average age of 25.3 years

For each subject, we had MRI sequences of images with 8echoes at 3T. The following images are example:

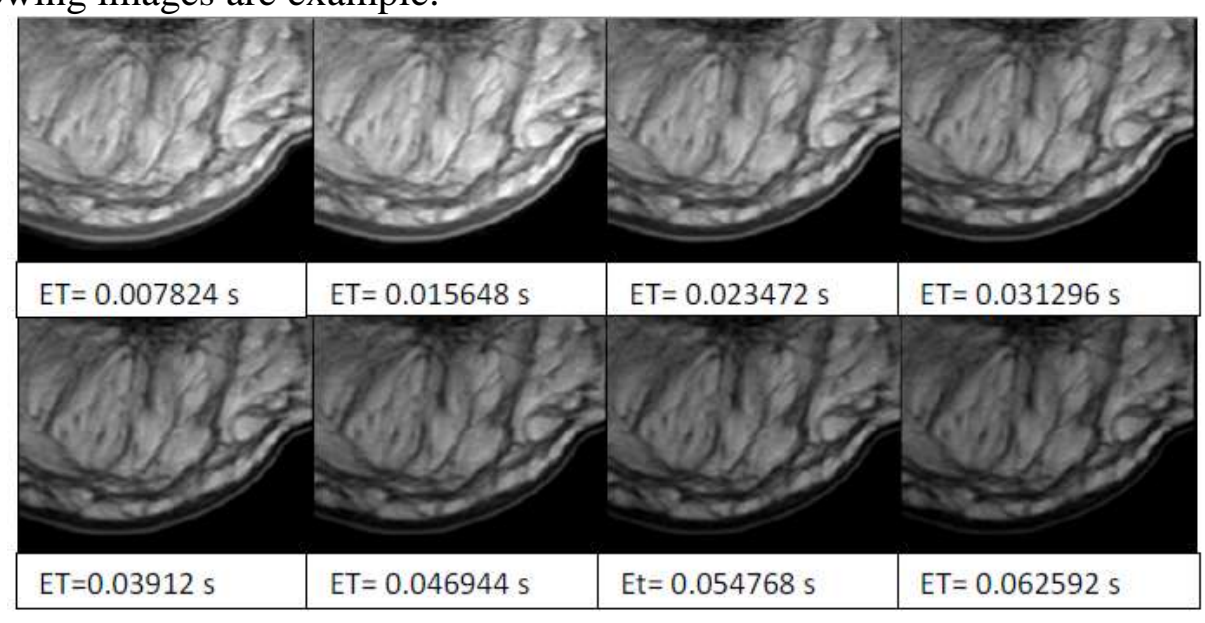

Figure2: MRI sequence: Turbo Spin Echo (TSE) calculation T2-weighted sequence.

The T2 calculation of TSE sequence using Fiji ImageJ gives these images as results: 


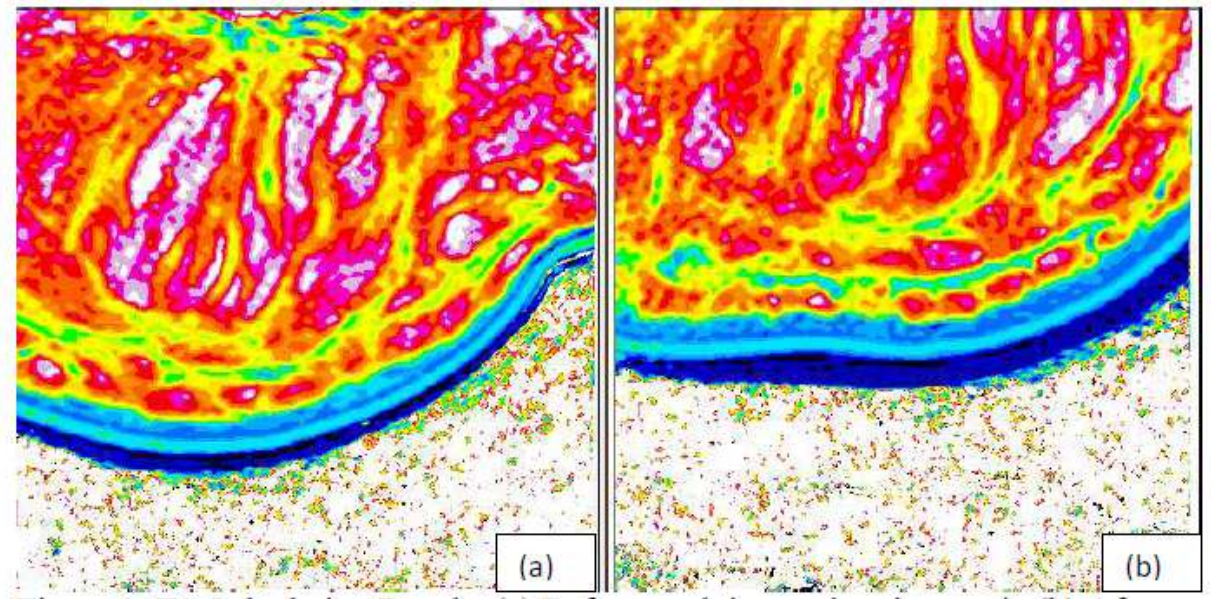

Figure 3: T2 Calculation Results (a) Before applying moisturizer topic (b) After application.

The application of Fuzzy C-means Clustering using MATLAB for 10 clusters gives these results:

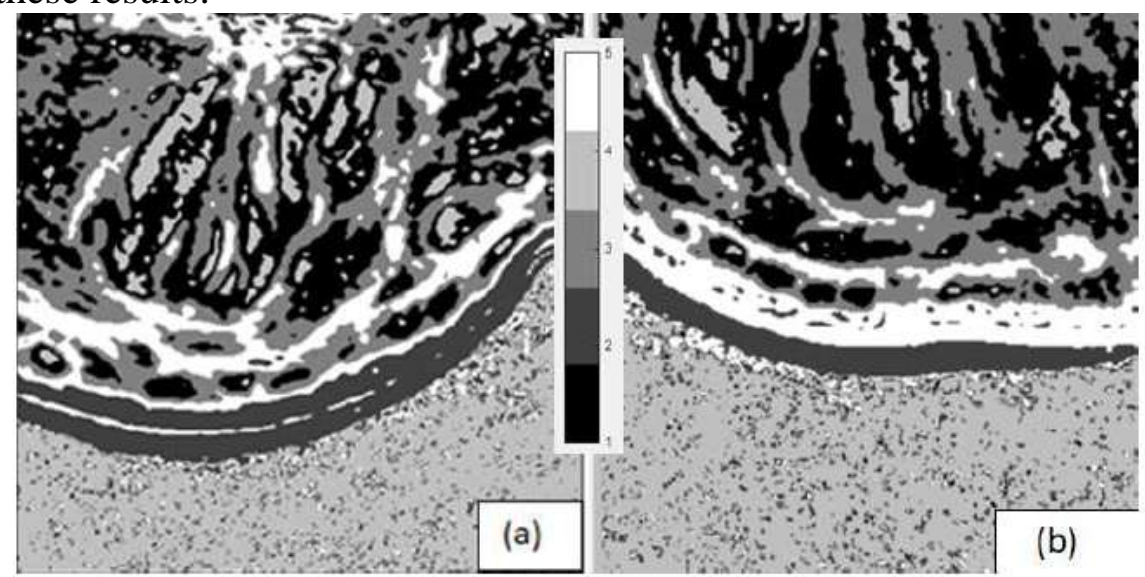

Figure 4: Results of FCM segmentation method; (a) Before applying moisturizer topic (b) After application.

This study involves applying FCM segmentation method for MRI data, to observe the effects of a topical moisturizer on the different skin layers. We can observe that the epidermis layer before applying the moisturizer topic is represented by different classes than after application.

We can conclude first that MRI makes an ideal imaging modality of unique soft tissue contrast to study the skin anatomy and to observe the different skin's layers. Then, the application of moisturizer topic provides effects in epidermis layer of skin, exactly in stratum corneum, which view with large area after the application of moisturizer topic.

\section{Conclusion}

The aim of this article is to review the segmentation approaches for Magnetic Resonance Imaging. We started by classifying these approaches, then briefly described Fuzzy C- means Clustering method. Furthermore, we presented the sample of our study with a discussion of the results obtained using FCM segmentation method. We conclude that MRI is an attractive modality to study the skin water content, it makes an ideal observation of the different skin layers in vivo 
with three dimensions. However, the segmentation of MRI data by FCM clustering is a computerized method to help clinicians in order to measure skin hydration.

\section{Conflict of interest statement}

Authors declare no conflicts of interest.

\section{Authors' biography}

Rachida ZEGOUR is currently a Ph.D. student in Medical Computing Laboratory (LIMED) at Mira Abderahmen university, Bejaia, Algeria. She obtained her Master's degree in 2015 from M'Hamed Bouguerra University, Boumerdes, Algeria. Currently, she is working on segmentation of medical images (MRI) using Fuzzy methods. Her main research interests include medical image processing and machine learning.

Dr. Ahror BELAID received the M. S. degree in Operation Research from University of Abderrahmane Mira, Bejaia, Algeria. He received his Ph.D. degree at the Department of Information Processing Engineering, Heudiasyc laboratory, Compiègne University of Technology, France. He is currently Associate Professor with the Department of Computer Science, Abderrahmane Mira University. His current research interests include Image Processing and Machine Learning.

Douraied BEN SALEM, MD, PhD, is Professor of Radiology at the University of Western Brittany (UBO, Brest, France) and member of the Laboratory of medical information processing - LaTIM, INSERM UMR 1101 (Brest, France). He received his medical and doctoral degree from the University of Burgundy (Dijon, France). $\mathrm{He}$ is Associate Editor of the "Journal of Neuroradiology" and member of the Editorial Board of "Heliyon" and of the "Journal of Forensic Radiology and Imaging".

\section{References:}

[1] Jawad Mesrar, Julien Ognard, Marc Garetier, DavidChechin, Laurent Misery and Douraied Ben Salem, In vivo skin moisturizing measurement by high resolution 3 Tesla magnetic resonance imaging, Skin Research and Technology 2017; 23: 289-294, doi: 10.1111/srt.12333. https://doi.org/10.1111/srt.12333

[2]Ivana Despotovic, Bart Goossens, and Wilfried Philips, MRI Segmentation of the Human Brain;Challenges, Methods, and Applications, Computational and Mathematical Methods in Medicine, Volume 2015 (2015), Article ID 450341, 23 pages http://dx.doi.org/10.1155/2015/450341. https://doi.org/10.1155/2015/450341

[3]J.A. McGrath, R.A.J. Eady \& F.M. Pope, Anatomy and Organization of Human Skin, textbook of dermatology $\quad 1-1-153, \quad 2010, \quad$ https://doi.org/10.1002/9780470750520.ch3. https://doi.org/10.1002/9780470750520.ch3

[4]Elisa de Oliveira Barcaui, Antonio Carlos Pires Carvalho, Juan Pi-eiro-Maceira, Carlos BaptistaBarcaui, and Heleno Moraes, Study of the skin anatomy with high-frequency (22 $\mathrm{MHz}$ )ultrasonography and histological correlation, Radiol Bras. 2015 Sep-Oct; 48(5): 324-329. doi:10.1590/0100-3984.2014.0028. https://doi.org/10.1590/0100-3984.2014.0028

[5]Lídia Palma, Liliana Tavares Marques, Julia Bujan, and Luís Monteiro Rodrigues, Dietary water affects human skin hydration and biomechanics, Clin Cosmet Investig Dermatol. 2015; 8: 413-421.

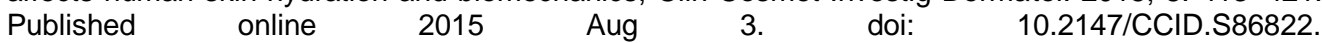
https://doi.org/10.2147/CCID.S86822

[6]Joëlle K. Barral, Neal K. Bangerter, Bob S. Hu, and Dwight G. Nishimura1, In Vivo High-Resolution Magnetic Resonance Skin Imaging at $1.5 \mathrm{~T}$ and $3 \mathrm{~T}$, published in final edited form as: Magn Reson 
Medical Technologies Journal, Volume: 2, Issue: 3, July-September 2018, Pages:257-265.

Doi :https://doi.org/10.26415/2572-004X-vol2iss1p255-261

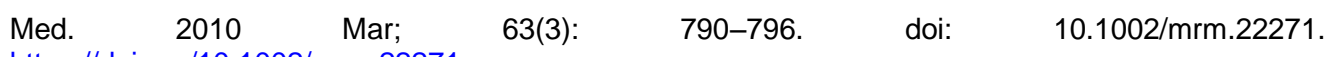

https://doi.org/10.1002/mrm.22271 cooperatives and Bayesian formulation, 29 October 2008

[8] Rafael C. Gonzalez and Richard E. Woods, Digital image processing, Forth edition, 2017

[9]Mahipal Singh Choudhry and Rajiv Kapoor, Performance Analysis of Fuzzy C-Means Clustering Methods for MRI Image Segmentation, Twelfth International Multi-Conference on Information Processing-2016 (IMCIP-2016).

[10] Yuhui Zhenga, Byeungwoo Jeond, Danhua Xua, Q.M. Jonathan Wua, and Hui Zhanga, Image segmentation by generalized hierarchical fuzzy C-means algorithm, Journal of Intelligent \& Fuzzy Systems 28 (2015) 961-973 DOI:10.3233/IFS-141378 .

[11] Keh-Shih Chuang, Hong-Long Tzeng, Sharon Chen, Jay Wu and Tzong-Jer Chen, Fuzzy c-means clustering with spatial information for image segmentation, Computerized Medical Imaging and

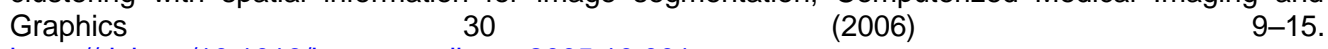
https://doi.org/10.1016/i.compmedimag.2005.10.001

PMid:16361080

[12] Mohamed N. Ahmed, Member, IEEE, Sameh M. Yamany, Member, IEEE, Nevin Mohamed, Aly A. Farag, Senior Member, IEEE, and Thomas Moriarty, A Modified Fuzzy C-Means Algorithm for Bias Field Estimation and Segmentation of MRI Data, IEEE TRANSACTIONS ON MEDICAL IMAGING, VOL. 21, NO. 3, MARCH 2002.

[13] Sudip Kumar Adhikari , Jamuna Kanta Sing, Dipak Kumar Basub, Mita Nasipuri, Conditional spatial fuzzy C-means clustering algorithm for segmentation of MRI images, Applied Soft Computing 34 https://doi.org/10.1016/i.asoc.2015.05.038

[14] Mohamed Baghdadi, NacéraBenamrane and Lakhdar Sais, Fuzzy generalized fast marching method for 3D segmentation of brain structures, Imaging Systems and Technology, Volume27, Issue3, September 2017,

Pages 281-306. https://doi.org/10.1002/ima.22233 